\title{
Digital creativity in dementia care support
}

\section{Konstantinos Zachos, Neil Maiden, Kristine Pitts and Sara Jones}

Centre for Creativity in Professional Practice,

City University London,

Northampton Square, London, EC1V0HB, UK

Fax: +44-20-7040-8427

E-mail: kzachos@soi.city.ac.uk

*Corresponding author

\section{lan Turner, Malcolm Rose and Kevin Pudney}

Registered Nursing Home Association, Birmingham B30 3JN, UK

Fax: +44-121-486-3175

E-mail: ian.turner@rnha.co.uk

\section{Julie MacManus}

\author{
MediaTel Ltd., \\ London W1B 5AJ, UK \\ E-mail: juliemacmanus@gmail.com
}

\begin{abstract}
This paper reports the application of digital creativity to the care for people with dementia. A digital app was developed for use by staff in residential homes caring for people with dementia. The app invokes a set of computational services to undertake creative work, the results of which are presented to encourage and support cognitive creativity by care staff. The services undertake computational work such as retrieving resolutions to care problems encountered previously, matching care situations to situations in analogical domains such as parenting, teaching and prison life, and automatically generating customised creativity prompts. The app was designed so that care staff can generate contributions to resident care plans that are novel and hence individualised to the resident as part of a shift towards more person-centred care.
\end{abstract}

Keywords: digital creativity; case-based reasoning; analogical reasoning; dementia care; mobile computing.

Reference to this paper should be made as follows: Zachos, K., Maiden, N., Pitts, K., Jones, S., Turner, I., Rose, M., Pudney, K. and MacManus, J. (2013) 'Digital creativity in dementia care support', Int. J. Creative Computing, Vol. 1, No. 1, pp.35-56.

Biographical notes: Konstantinos Zachos received his Computer Science Diploma degree from RWTH Aachen in 2004 and his PhD in Software Engineering (specialisation in service-oriented requirements engineering and creativity) from City University London in 2008. He is a Researcher at the 
Centre for Human Computer Interaction Design at City University and has been involved in research through numerous EU-funded projects in the areas of requirements engineering, service discovery mechanism and techniques, and creative problem-solving and learning.

Neil Maiden is a Professor of Systems Engineering and a co-founder of the Centre for Creativity in Professional Practice at City University London. His research interests include software engineering, creativity in design thinking, and creativity support tools. He has over 150 peer-reviewed journal and conference publications and been a principal investigator on research grants with a total value of over $£ 3$ million. He has served on many editorial boards including IEEE Transactions on Software Engineering and IEEE Software, and was editor of the IEEE Software Requirements column from 2005 to 2013.

Sara Jones is a Senior Lecturer with expertise in creativity and the design of interactive systems. From 2006-2011, she held a RCUK research fellowship in creativity applied to design and engineering and has led six funded research projects in these areas. Recent research projects include an investigation of creative engagement with visualisations of data from smart homes for a major energy provider, investigation of information spaces for creative conversations in design education, and the EU FP7-funded MIRROR project. She currently teaches creativity in interactive system design and is Programme Director for the innovative Masters in Innovation, Creativity and Leadership.

Kevin Pudney was the Head of Information and Communication Technology for the Social Services Department of a major English county. With a background in research methods, and qualified in Prince2 and ITIL (IT Infrastructure Library), he has wide experience in the development and implementation of electronic systems, including business process reengineering, in social care settings. His last major project was recognised in the British Computer Society awards for 2005 for the successful implementation of a web-based, multi-agency, electronic social care record (ESCR) system for 3,000+ users. A particular interest is in exploring how mobile devices can be harnessed to improve care.

Malcolm Rose is an Applied Economist who has worked at influential levels in local and regional government in policy, European affairs and regeneration. A former Industrial Fellow at the University of Nottingham's Institute for Enterprise and Innovation he has worked with both academic and practitioner experts on a range of EU-funded projects. In the past decade, he has been particularly concerned with the implications of the ageing population and how they will be cared for.

Ian Turner holds a degree in Pure and Applied Mathematics and worked in a variety of technical and marketing roles for International Computers Ltd (now part of Fujitsu) in the 1970s and 1980s. He has owned and managed nursing homes since 1985 and now runs six care homes in East Anglia providing care for some 300 residents. He chairs the Registered Nursing Home Association that supports some 1,000 member homes by providing services such as disclosure and barring checks. He sits on a variety of committees for the Department of Health and Skills for Care.

Julie MacManus graduated from City University's Human-Centred Systems MSc in 2011 where she developed an interest in creative problem solving in computing. Her dissertation ran in parallel to the MIRROR project, exploring ways in which creative problem solving could intervene in the care of people with dementia and introduced some techniques to care workers. She is currently in charge of product development and client services at a leading online media business intelligence service. 


\section{Dementia care and digital creativity}

Dementia is a condition related to ageing. After the age of 65 the proportion of people with dementia doubles for every five years of age, so that almost one fifth of people over the age of 85 and a third of people over the age of 95 are estimated to be affected (Alzheimers Society, 2010). There are currently 750,000 people in the UK with dementia, a figure projected to double by 2051 whenit will affect a third of the population either as a sufferer, relative or member of care staff (Knapp and Prince, 2007). Worldwide the number of people with dementia is estimated at 35 million, a figure also expected to double in 20 years (Wimo and Prince, 2010). Two-thirds of current care home residents in the UK have a form of dementia (Knapp and Prince, 2007), and caring for them poses complex and diverse problems for care staff due to the different needs of individual residents. Moreover, care needs to be delivered within constraints imposed by residential care homes - constraints such as limited time and spaces for co-located creative problem solving.

A more person-centred care of people with dementia seeks an individualised approach that recognises the uniqueness of each resident (Brooker, 2007). We conjecture that customising dementia care to individuals necessitates creative thinking to produce outcomes that are both useful and novel (Sternberg, 1999). Therefore, a creative plan should not only improve the care of the resident, but also be new to the care of the individual resident, and/or perhaps also be novel to the individual care staff and/or care team. However, there is currently little explicit use of creative problem solving in dementia care. Indeed, low status, poor pay and high staff turnover (All-Party Parliamentary Group on Dementia, 2009) mean that care staff have at best limited training in creative problem solving.

In the remainder of this paper, we report a digital creativity app and the research undertaken to design it. In the next section, we outline current dementia care challenges and observed opportunities for creativity to deliver the more person-centred care agenda. We then describe the mobile digital app and three of the computational services that it invokes to undertake and support the creative work needed to enhance the care plans of individual residents. The paper ends with a review of related work and plans to evaluate the app in residential care practices.

\section{Challenges caring for people with dementia}

People with dementia experience difficulties understanding their environment. They operate in their own version of reality that is often hard for care staff to interpret (Alzheimers Society, 2010). As a consequence caring for people with dementia is complex, and care staff are expected to have detailed knowledge of the ageing process, skills to encourage older people and families to participate in care, and capabilities to meet a wide range of needs associated with nutrition, in continence and mental health (Fitzpatrick and Roberts, 2004).

However, caring for people with dementia is also one of the lowest paid UK occupations (Machin and Wilson, 2004], and care staff turnover at 20\%-23\% per annum (All-Party Parliamentary Group on Dementia Report, 2009) is a major hindrance to developing a skilled workforce. The problem is compounded by minimum training to 
care staff arising from a lack of regulations for training that dementia care staff should receive. This low status and lack of training pose barriers to the adoption of successful creative problem solving in dementia care, although making the role more creative could raise its status and pay.

In response to these challenges, modern care practices have evolved. For example, the Bradford Dementia Group has altered how dementia and the provision of care services are conceptualised (Baldwin and Capstick, 2007). It introduced the notions of person-centred care and personhood in relation to people with dementia, driving forward a more user-centred approach to care delivery. The four elements of person-centred care are:

1 a value base that asserts the absolute value of all human lives

2 an individualised approach that recognises the uniqueness of each resident

3 understanding the world from the perspective of the service user

4 providing a social environment that supports psychological needs (Brooker, 2007)

Dementia care mapping identifies areas for care improvement through detailed, skilled and empathic observation of a person with dementia's activities and interactions with staff. The aim is to provide the person with dementia's point of view. The emerging focus on more person-centred care suggests a greater role for creative problem solving that delivers an individual approach to maintain each resident's sense of identity.

\subsection{Creative problem solving in nursing care}

Creative problem solving is not new to nursing. Osborn (1965) reported that creative problem solving courses were introduced in nursing and occupational therapy programmes in the 1960s. Le Storti et al. (1999) developed a programme that fostered the personal creative development of student nurses, challenging them to use creativity techniques to solve nursing problems. This required a shift in nursing education from task-to role-orientation and established a higher level of nursing practice - a level that treated nurses as creative members of healthcare teams. Arbesman and Puccio (2001) also put the case for creative problem solving by nursing administrators who set the tone in a work unit, and how others undertake creative work. Houts et al. (1996) proposed a prescriptive creative problem-solving model to help family carers deliver care to people with chronic diseases discharged from hospital. The model was tailored to the healthcare domain from the Osborn-Parnes creative problem solving model (Osborn, 1965). It distinguished situations in which creative problem solving could be used from situations in which advice needs to be sought from experts. Case studies were used to teach care staff a process in which they adapted solutions developed for other people to family members in their own care.

\subsection{Calls for creative practice in care homes}

Previously there have been calls for creative approaches to be used in the care of people with dementia. Successful creative problem solving might counteract the negative and stressful effects that are a frequent outcome of caring for people with dementia [Help the Aged, 2007). Several dementia care learning initiatives are creative in their approach. 
One is the adoption of training courses in which care staff are put physically into residents' shoes (Davis, 2010). Another is the use of guided exercises in which participants are encouraged to experience life mentally through the eyes of someone with dementia (Brooker, 2007). In particular caring for people with late stage dementia is recognised to require more creative approaches. Interventions such as Snoezelen provide sensory stimuli to stimulate the primary senses of sight, hearing, touch, taste and smell. A common theme is the need to adapt to an individual's behavioural patterns and habits based on life histories.Overall however, in spite of these initiatives, there has been little systematic use of creative problem solving to individualise dementia care.

\subsection{Opportunities for creative problem solving in dementia care}

To discover opportunities for creative problem solving in dementia care, we collaborated with one care home in the east of England rated as good by the UK's Care Quality Commission (CQC). The home acted as a pilot site. Full details are reported in MacManus (2011). Observations and interviews with care staff in the home revealed three distinct opportunities for creative problem solving in dementia care:

1 Managing challenging behaviour: care staff can encounter challenging resident behaviour such as the refusal of food or medication, and verbal or physical aggression. Creative problem solving can be applied to enhance the care plan for the resident to resolve such behaviour. For example, if a resident is uncooperative with care staff when taking medication, one enhancement to his care plan might be to require care staff to wear a doctor's coat when providing the medication. The enhancement is creative because it can be useful, novel to the resident if not applied to him before, and perhaps novel to the care team who have not applied this plan in the home beforehand.

2 Adapting to an evolving understanding of each resident: understanding a resident is the major challenge in dementia care, and best achieved through reflection on the resident's past and reactions to the present. Something new is learned every day, so care staff needs to think creatively in response to new evidence to understand a resident more completely. For example, when a resident began to trash his room, overturn mattresses and tip out the content of drawers, care staff could not resolve this situation. Stopping him caused him to become agitated. However, understanding the resident's past career as a prison guard provided a clue to this behaviour, so one care plan enhancement might be to allow him to finish each 'search' before intervening. Again, this enhancement can be considered creative because it is useful and novel to the resident, as well as perhaps novel to the care team.

3 Devising stimulation to residents with advanced stage dementia: most residents at the care home have advanced dementia, which makes engaging them in activities challenging. Creative thinking offers a means to discover new forms of stimulation through all five senses for these residents within one major constraint - limited staff time. For example, a fragrance-release system to stimulate appetite among people with dementia releases three food fragrances a day to coincide with the user's mealtimes. It is less stigmatising than an alarm or reminders to eat. Again the enhancement might be creative because it is useful and new to the resident, as well as perhaps novel to the care team. 
The next section reports an exploration of different creativity techniques with staff in the home to explore those have the potential to deliver one or more of these opportunities.

\subsection{Creativity techniques to adopt in dementia care}

One half-day creativity workshop was held with one activity coordinator, one nurse and four senior care assistants from the pilot home to improve decision-making, manage challenging behaviours and devise stimulations. It was led by one of the authors. Different creativity techniques were used in a three-stage process to understand a fictional situation, generate ideas for it and prepare for action. The techniques were described to the care staff as problem solving techniques to stress their practical relevance, and were:

- lotus blossom (Michalko, 2006)

- what-if(Innovation Company, 2002)

- brain-writing (Higgins, 2006)

- other worlds (Innovation Company, 2002)

- what/how (Higgins, 2006).

Full details of the use of the techniques are in MacManus (2011).

The session revealed an appetite and potential the other worlds technique. Other worlds is an exploratory creativity technique that encourages similarity-based reasoning across domains. During the workshop the care staff sought to generate ideas to resolve care problems in four different, less constrained domains - social life, research, word of mouth and different cultures. These ideas were then transferred to the care domain to explore their effectiveness in it. The staff also randomly picked a fifth analogical domain from politics, tennis, flying and army and repeated the idea generation process.

The other worlds technique was judged to be the most effective as well as the most interesting to staff. It created more ideas than each of the other techniques, and two of the ideas from the session were deemed sufficiently novel and useful to implement in the care home immediately. In particular, care staff valued how it could be applied to make knowledge and ideas from experts and outside of the dementia care domain. It became the focus of our delivery of creativity support.

\subsection{Towards a digital creativity app}

Effective use of the other worlds technique in creative problem solving normally requires both facilitated guidance to explore the different worlds and communication between participants to share ideas. Neither was possible on a regular basis in constrained care home settings. Therefore, we decided to implement digital support for the technique in the form of an app. In the place of human facilitation, the app would automatically retrieve then guide care staff to explore concrete other worlds. And in place of face-toface communication, the app would automatically support the asynchronous communication between care staff who would digitally share information about care ideas and practices via the app. 
The app supports three forms of the other worlds technique using three different computational services that the app invokes:

1 to generate ideas in a different world with different constraints, the app can invoke a service to recommend other worlds to explore

2 to exploit ideas generated previously in different worlds, the app can invoke a service to retrieve successful resolutions to challenging behaviours in other domains such as parenting and prisons

3 to exploit ideas generated previously in other residential care homes, the app can invoke a service to retrieve successful resolutions to challenging behaviour exhibited by residents in these homes.

Indeed, senior staff and staff in specialised services are known to reuse strategies to resolve problem situations, but these resolution strategies are not accessible to many of the less-experienced 1.6 million care staff in England. Therefore the services support the creative reuse of care enhancement plans shared digitally across residential homes.

Moreover, we observed that care staff in the pilot home maintained electronic resident records on a small number of desktop PCs - a common situation in most residential care homes in the UK. However, this meant that care staff only accessed and entered resident data at the start and end of shifts, which in turn caused queuing and rushed data entry. A desktop-based digital solution would not fit with working practices. Therefore, we initially replaced the paper notes of care staff in the pilot home with mobile iPod Touch devices running apps to capture and share observations about residents (Karlsen et al., 2011). The pilot was successful, so we designed a new app to run on this device and be used by care staff in quieter moments during their shifts. The app was named Carer, and is described at length in the next section.

\section{The Carer app}

We designed Carer to be used with minimum user training or management overhead. Care staff describe new situations that they encounter and cases of good care practice in unrestricted natural language. A typical example of a care situation that a member of care staff might enter into app is:

Mrs. $X$ acts aggressively towards care staff and the resident verbally abuses other residents at breakfast. Suspect underlying insecurities to new people.

Carer delivers creativity support with the components shown in Figure 1:

1 a mobile app that care staff interact with

2 a digital repository of descriptions of good care practices

3 two services to retrieve cases

4 one service to generate other worlds

5 one service to generate creativity prompts. 
The mobile app passes a situation input by care staff to a discovery service that retrieves an ordered set of matched case descriptions that care staff can browse and select, then request creativity triggers to guide more focused creative thinking.

Figure 1 The Carer architecture, showing how digital sharing of knowledge about good practices related to care is used by computational creativity services to deliver support to care staff (see online version for colours)

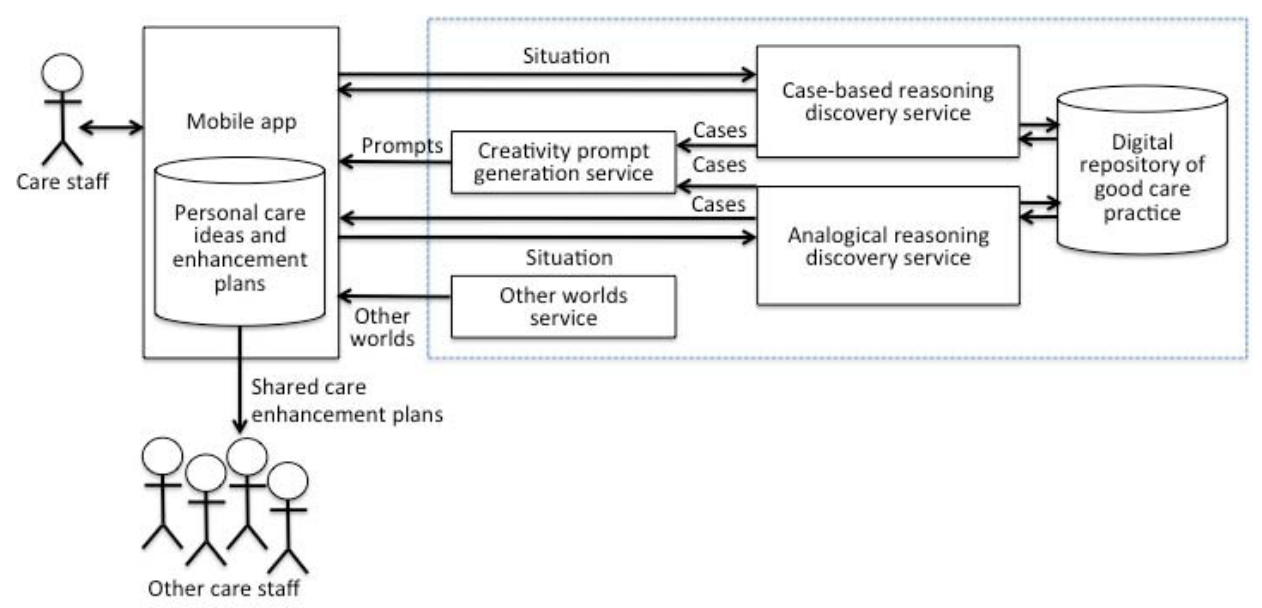

The architecture was designed to support different forms of creative thinking with the other worlds technique using four computational creativity services:

1 the other worlds service automatically generates different domains such as travelling or cooking in which to generate care plan enhancements to a current situation

2 the analogical reasoning discovery service computationally matches a current situation to good care practices from non-care domainssuch as parenting and prisons

3 the case-based reasoning discovery service computationally matches a current situation to good care practices from the residential care sector based on reported good care practices (Houts et al., 1996)

4 the creativity prompt generation service automatically generates statements from retrieved good care practices that care staff can use to generate new ideas about care enhancement plans.

\subsection{The mobile app component}

The app that care staff use to describe care situations, browse retrieved cases and generate ideas and care plan enhancements is an iOS app designed to run on iPod Touch and iPhone devices. A user enters the natural language description of a care situation into the text box on the left-hand side of Figure 2 using the device's keyboard, then selects whether to receive cases from the care domain or to receive analogical cases from other domains. The user can then browse the names and keywords of retrieved cases to select the ones to work with further, as shown on the right-hand side of Figure 2. 
Figure 2 Describing a care situation and receiving care cases retrieved by the case-based reasoning service in Carer (see online version for colours)
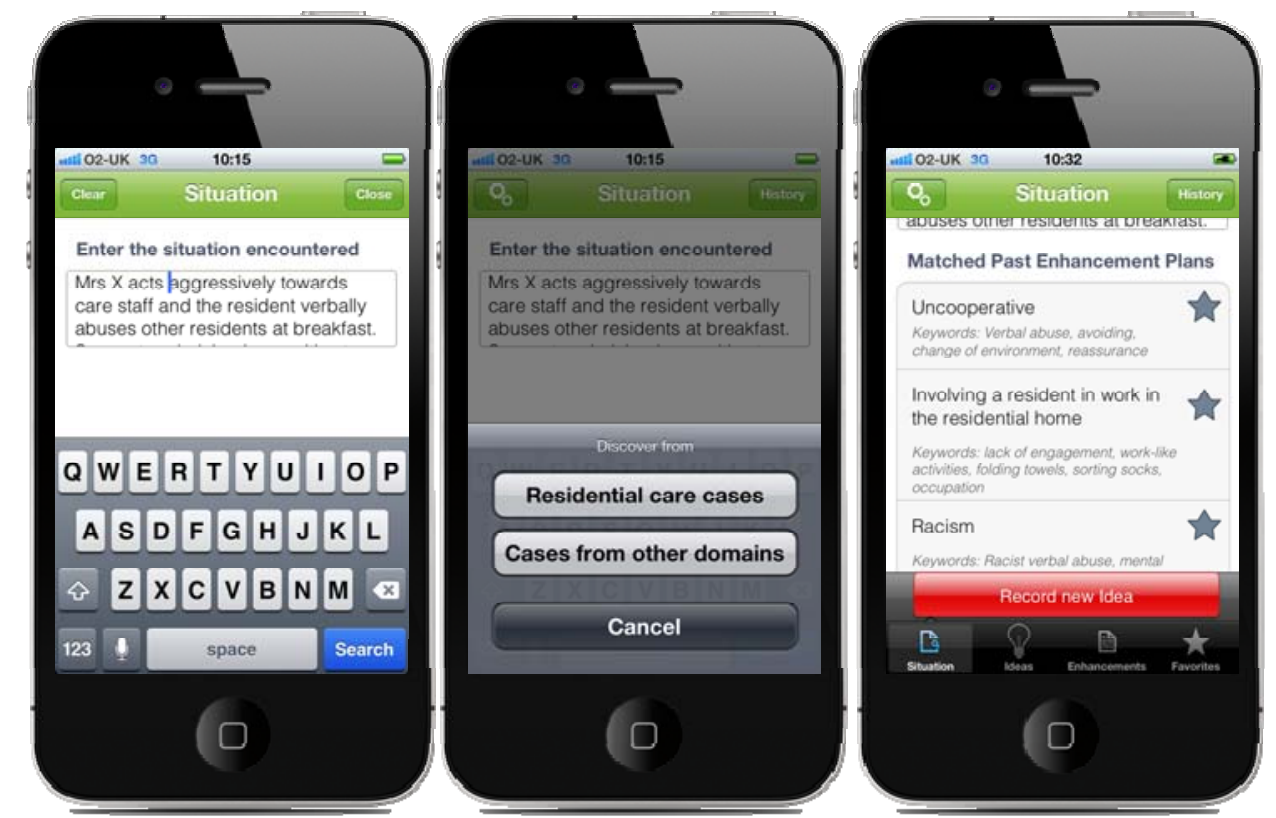

Figure 3 shows how users can read each selected case. Each case is described and presented in text form in two parts - the situation that was encountered and the care plan enhancement that was successfully applied - that the user is able to scroll between. The left-hand side of Figure 3 shows one case retrieved for our example situation in the care domain - it described how care staff elsewhere successfully resolved a situation with an uncooperative resident. The right-hand side shows a case retrieved using the analogical matching discovery service. The case is taken from the policing domain, and describes plans that were successfully implemented to handle challenging situations when arresting drunken people.

Care staff can then seek to generate new ideas directly from the retrieved case descriptions. However, formative usability evaluations revealed that the dense text format does not always support this effectively. Furthermore, just presenting care plan enhancements applied to other residents might not encourage the more creative person-centred care (Baldwin and Capstick, 2007) that we were seeking. Therefore, care staff can request the app to generate prompts for creative thinking automatically from the case. Each request generates two concise creativity prompts with which to generate ideas - examples of pairs of prompts generated from the case presented on the left-hand side of Figure 3 are shown on the left-hand side of Figure 4.The prompts include think about how to give her reassurance and understanding, and think about how not to take it personally. A different pair of creativity prompts can be requested by simply flicking the iPod screen. This gives the user simple and rapid access to a large number of creativity prompts in a short time. 
Figure 3 Cases descriptions retrieved by the case-based reasoning discovery and analogical matching discovery services, and presented by the app (see online version for colours)
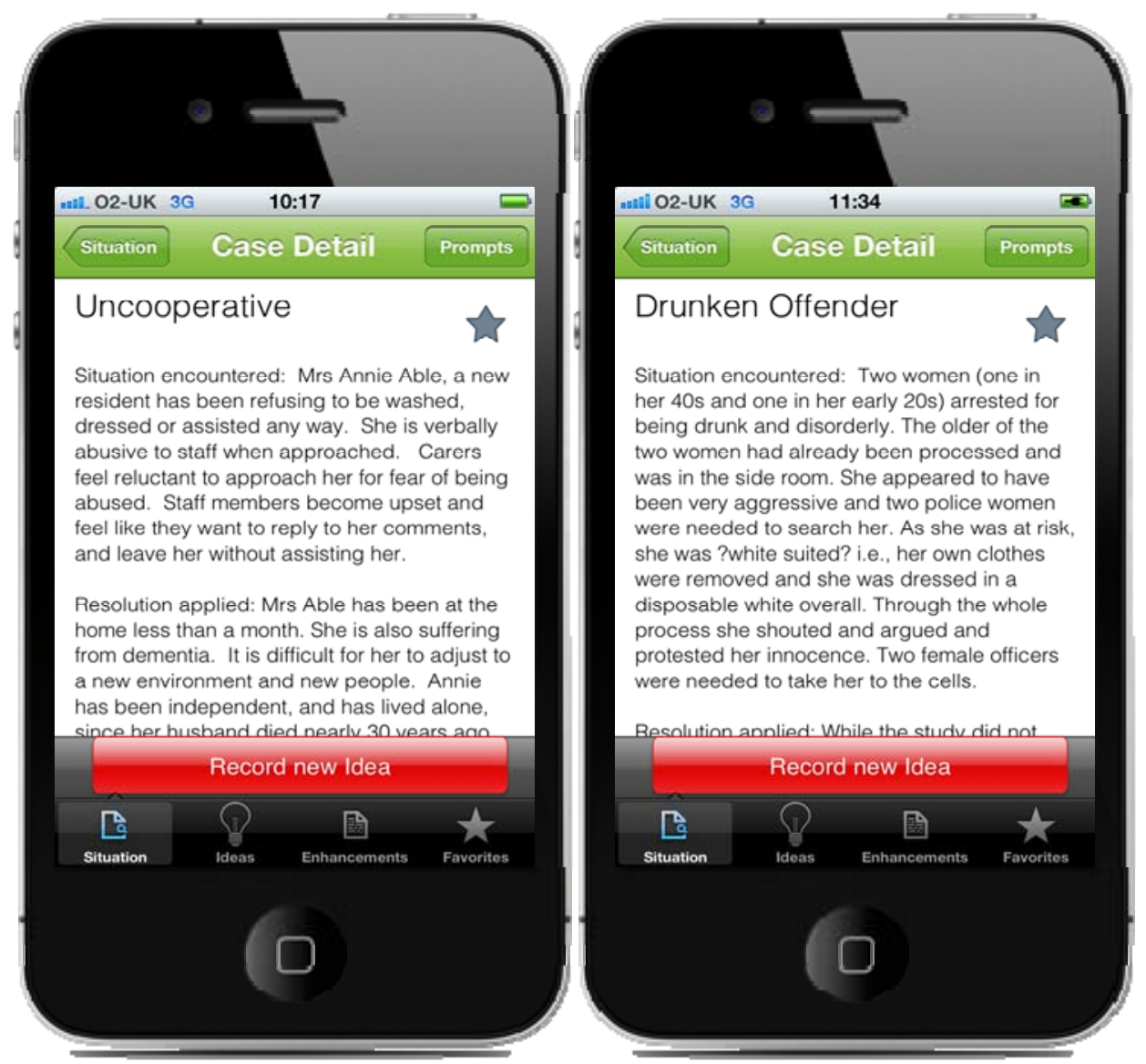

Furthermore, at any time, care staff can also explore different other worlds in which to generate new ideas without the constraints of the care domain. An example of this guidance is shown on the right-hand side of Figure 4. The user is encouraged to think about how to solve the aggression situation as a waitress on a busy Saturday night. Again, a simple flick of the screen will generate a different other world, such as parachuting from an aircraft.

Care staff can record new ideas in audio form at any time by simply pressing the red button visible in Figures 3 and 4, verbalising the idea, then using the device's keypad to give it a meaningful name. Using this feature, care staff can quickly build up a set of ideas with which to generate different care plan enhancements. The use of idea audio recording was adopted to overcome possible barriers to creative flow imposed by touch 
screen keyboards. It also allows care staff to play back and share ideas more effectively to encourage reflective learning, similar to the use of the audio channel in digitally-supported creative brainstorming (van Dijk et al., 2011).

Figure 4 Example creativity prompts generated by the creativity prompt generation service and presented by the app (see online version for colours)
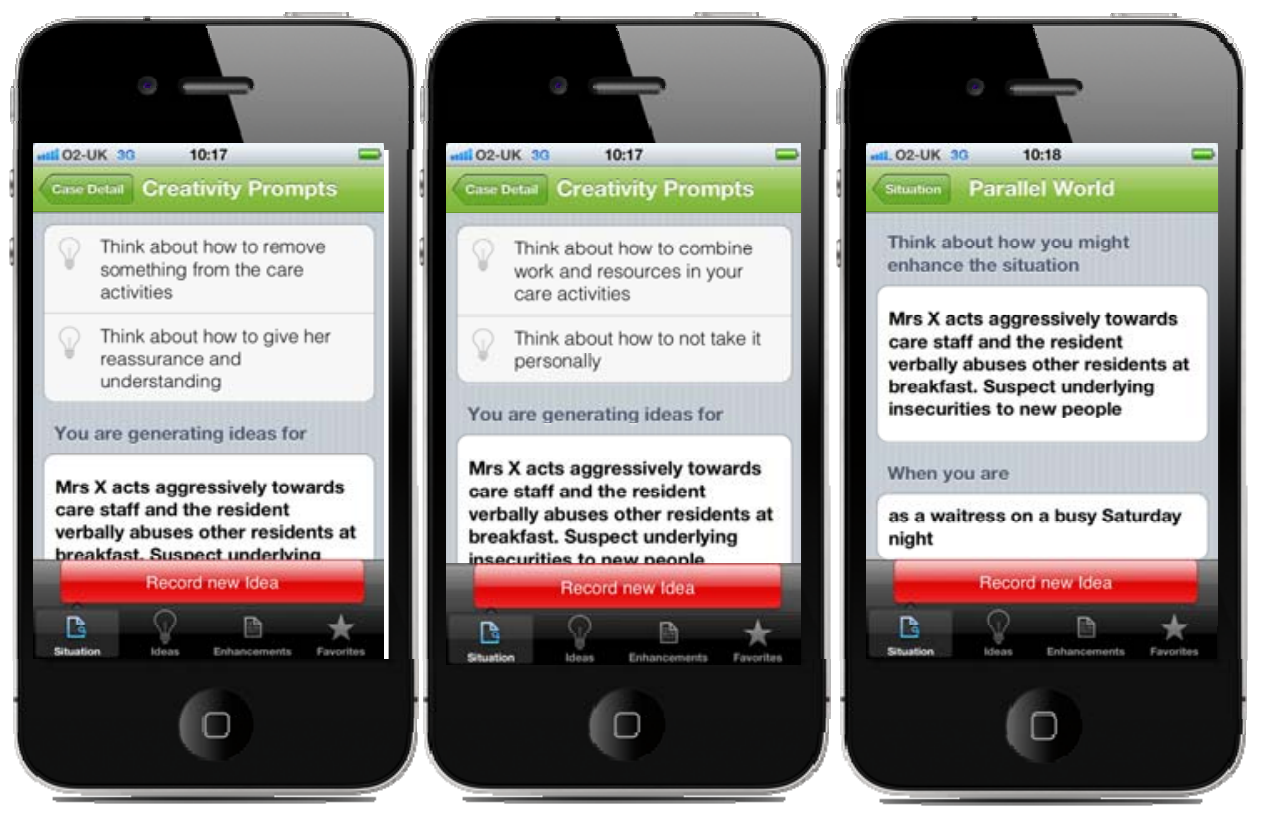

Care staff can also, at any time, edit and manage their pool of ideas, as shown on the lefthand side of Figure 5. They can also select and order these ideas to construct a new care enhancement plan. The example plan on the right-hand side of Figure 5 shows a plan composed of three ideas and one comment: the plan orders the ideas in their sequence of implementation - to understand reasons for verbal abuse, then understand the situation from the perspective of the resident, then collect personal items to make her more at home. A text comment added to the plan suggests generalising the plan to all new residents.

Another important app feature is plan playback. This feature was implemented to allow the user to reflect on the plan, revise it and extend it with more ideas and comments before the new care is given to the resident. And to support collaborative reflection with colleagues over the plan, Carer enables the user to share the plan as e-mail attachments and/or in a private micro-blogging network implemented to increase digital communication between care staff. 
Figure 5 A care enhancement plan constructed from ideas to resolve the care situation described in Figure 2 (see online version for colours)

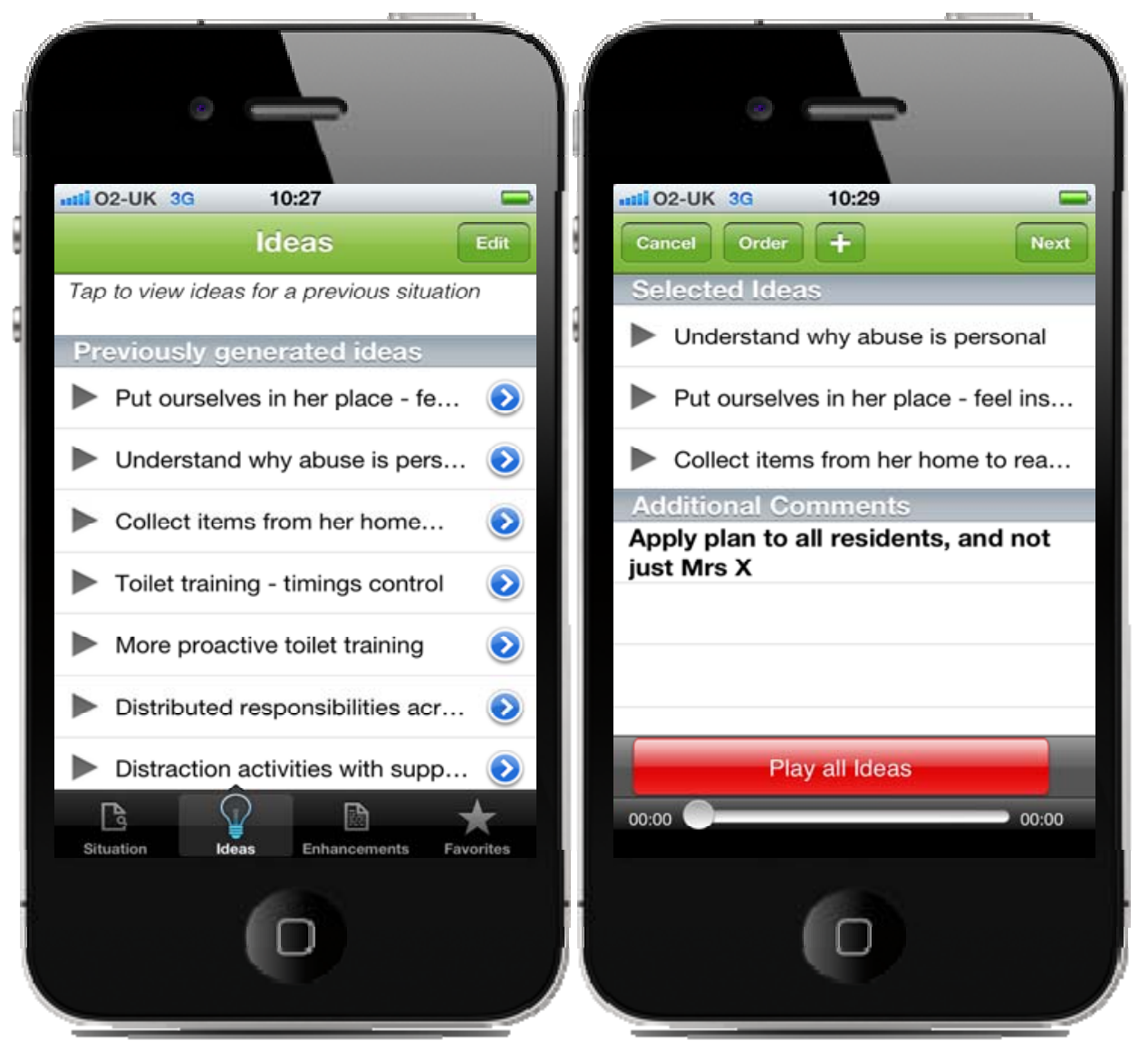

\subsection{A digital repository of care practice cases}

A digital repository stores natural language descriptions of cases of good care practice in XML based on the structure of dementia care case studies reported by the Social Care Institute for Excellence (Owen and Meyer, 2009). It is implemented using eXist, an open source native XML database featuring index-based XQuery processing that the discovery service queries using XQuery, a query language designed for processing XML data. Each case has two main parts - the situation encountered and the care plan enhancement applied - and is attributed to one class of domain to which the case belongs. No ontology or tags are used to provide additional semantic information about each case: the lack of resources or expertise to do this is not available in most UK residential care homes.

The current version of the repository stores almost 100 care cases. Almost 70 of these cases are cases of good practice in residential and dementia care. They are extracted from an online database of published good care practices at the Social Care Institute for Excellence (Owen and Meyer, 2009), elicited from focus groups with care staff, and provided directly by individual care staff wishing to share their good practices. The remaining 30 cases describe reported practices to enhance people's quality of life and 
overcome challenging behaviour situations in domains ranging from parenting and the pastoral care for students to commuting behaviour and prison life, and were collected from reports and from focus groups held with practitioners in these domains.

In the next three sections we report three of the computational creativity services implemented to exploit this case content in order to enable care staff to think more creatively about resident care. Each service was designed to compute this content within the constraints imposed by open-ended natural language descriptions of situations and cases.

\subsection{The case-base reasoning discovery service}

Although use of natural language can enable care staff to use the app with minimal training, the entered descriptions can be incomplete and ambiguous. Therefore, Carer uses information retrieval techniques to disambiguate each query term with its correct meaning then extend the query with extra terms with similar meanings to increase matches to terms in descriptions of past cases. Consider the example query in Figure 2. It is incomplete because the nature of the observed aggressive behaviour is not defined and ambiguous because insecurities could have different meanings such as the state of being subject to danger or injury, or the anxiety you experience when you feel vulnerable and insecure. Therefore the service, one version first built to solve software reuse problems (Zachos et al., 2007), delivers two capabilities:

1 Term disambiguation - select the meaning, or sense of each term in the situation to make it unambiguous.

2 Query expansion - add terms in the query fired at the case repository that have the same or similar meaning to existing query terms, to make the query more complete.

These capabilities are implemented in four components. In the first, the situation description is divided into sentences then tokenised and part-of-speech tagged and modified to include each term's morphological root (e.g., acted to act, residents to resident) using the Brill Tagger (Brill, 1992). In the second, the service applies procedures to disambiguate each term by defining its correct sense and tagging it with that sense by iteratively using context knowledge from other terms in the query (e.g., defining a resident to be a someone who lives at a particular place for a prolonged period rather than $a$ who lives in a hospital and cares for hospitalised patients under the supervision of the medical staff of the hospital). In the third, the service expands each term with other terms that have similar meaning according to the tagged sense, to increase the likelihood of a match with a case (e.g., the term aggressive is synonymous with the term hostile which is also then included in the query). In the fourth, the service matches all expanded and sense-tagged query terms to a similar set of terms that describe each case in the repository. Query matching is in two steps:

1 XQuery text-searching functions to discover an initial set of case problem descriptions that satisfy global search constraints

2 traditional vector-space model information retrieval, enhanced with WordNet, to further refine and assess the quality of the candidate case set.

The output is a ranked list of matched cases. 
The WordNet on-line lexicon fulfils an important role. It is a lexical database inspired by psycholinguistic theories of human lexical memory. It divides the lexicon into four categories: nouns, verbs, adjectives and adverbs. Word meanings, called senses, for each category are organised into synonym sets (synsets) that represent concepts, and each synset is followed by its definition or gloss that contains a defining phrase, an optional comment and one or more examples. It is also structured using semantic relations between word meanings that link concepts. WordNet's word senses and definitions provide the data with which to disambiguate terms in queries, and its semantic relations link terms to other terms with similar meanings with which to make queries more complete.

\subsubsection{Word sense disambiguation}

Assigning the correct sense to a word in context requires syntactic, semantic and pragmatic knowledge about the word, its part of speech and its context (Stevenson and Wilks, 2001). Therefore the service applies procedures to disambiguate senses using these knowledge types when more than one sense of a term is possible. Two of these procedures find query terms that are semantically connected to already-disambiguated terms, so that the new term is tagged with the same meaning as the connected term. A third assigns the most frequent sense to a term irrespective of its context (Wilks and Stevenson, 1996) - it had been used previously to baseline word sense disambiguation systems (McCarthy et al., 2004), and its performance is due to the skewed frequency distribution of word senses. A fourth procedure uses context-based senses, in which the SemCor bigrams method forms two pairs, one with the previous word, the other with the next word, and searches for these pairs in SemCor corpus (Miller et al., 1990). If in all of the occurrences of these pairs, the given word has the same sense, and the number of occurrences is bigger than a preferred threshold, then we assign that sense to the word. For example the algorithm produces a first version of the XML query with the noun terms resident, breakfast, insecurity and people and the verb terms act and suspect. Each term needs to be disambiguated and assigned a sense. Therefore the service assigns WordNet sense \#2 to the term act - behave in a certain manner; show a certain behaviour; conduct or comport oneself.

\subsubsection{Query expansion}

Next the service uses ontological information from WordNet to extract semantically related terms with the disambiguated senses using three methods:

- Synset expansion: terms are replaced by their synsets, for example the term act is replaced with its synset for sense \#2 [act, behave, do].

- Hypernym expansion: terms are augmented by their WordNet direct hypernyms, for example the hypernym of insecurity is anxiety.

- Gloss words expansion: terms are augmented with the terms in their glosses, for example the sense\#1 definition of the term insecurity is the anxiety you experience when you feel vulnerable and insecure. Hence, anxiety, vulnerable and insecure are extracted. 


\subsubsection{Query matching}

The expanded query is transformed into a XQuery that is fired at the situation descriptions of cases in the digital repository. Once an initial set of cases has been retrieved, a traditional vector-space (Salton et al., 1975) model information-retrieval step, enhanced with WordNet, is applied to refine and extract the most similar cases from the set. In the traditional vector-space model, documents and queries are represented as $\mathrm{T}$-dimensional vectors, where $\mathrm{T}$ is the total number of distinct words in the document collection after pre-processing. Each term in the vector is assigned a weight that reflects the importance of a word in the document. This value is proportional to the frequency a word appears in a document and inversely proportional to number of documents in which this word appears. Matching scores of original terms are assigned twice the weight as matching scores of expanded terms (synonyms, hypernyms, gloss terms). A higher overall score indicates a closer similarity between the query and case.

At the end of each invocation, the service returns an ordered set of the descriptions of the three highest-scoring cases for the app component to display as shown to the care staff in Figure 3.

\subsection{The analogical matching discovery service}

This service matches a description of an encountered situation to descriptions of cases of good care practice in other domains, for example good policing practices to manage disorderly revelers and good teaching practices to manage disruptive children. It approximates analogical similarities between two pieces of text based on the structuremapping theory (SMT) of analogy (Gentner, 1983), which seeks to transfer a network of related facts rather than unrelated one from a source to a target domain. A first version was implemented to support software service reuse (Zachos and Maiden, 2008). Again, the service can match situations and cases that use different terms to describe analogically similar problems.

To enable structure-matching, the service transforms natural language queries and case descriptions into predicates that express prepositional networks of nodes (objects) and edges (predicate values). Attributional predicates state properties of objects in the form predicate value (object) such as asleep (resident) and absent (relative). Relational predicates express relations between objects as predicate value (object1, object2) such as abuse (resident, care-staff) and remain(resident,room). According to the SMT, a literal similarity is a comparison in which attributional and relational predicates can both be mapped from a source to a target. In contrast an analogy is a comparison in which relational predicates but few or no attributional predicates can be mapped. Therefore, the service retrieves cases with high match scores for relational predicates and low match scores for attributional predicates, for example a match with the predicate abuse (detainee,police-officer) but no match with the predicate drunk (detainee).

To compute relational and attributional predicates the service invokes VerbNet and the Dependency Thesaurus as well as WordNet. VerbNet (Kipper et al., 2000) is a domain-independent verb lexicon that organises terms into verb classes that refine Levin's (1993) classes and adds sub-classes to achieve syntactic and semantic coherence among members of a verb class. The service uses it to expand query predicate values with different members from the same verb class. The Dependency Thesaurus supports dependency-based word similarity matching to detect similar words from text in a 
64-million word corpus (Lin, 1998). Given an input word it can automatically retrieve and cluster similar words. The service uses it to compute relational similarity between two sets of predicates.

When invoked, the service first divides the situation and case problem description texts into sentences, then part-of-speech tagged, shallow parsed to identify sentence constituents and chunked in noun phrases. It then applies 21 syntax structure rules and seven lexical extraction heuristics to identify predicates and extract lexical content in each sentence. Natural language sentences are presented as predicates in the form predicate value (object1, object2). The service then expands each query predicate with additional predicate values that have similar meaning according to verb classes found in VerbNet to increase the likelihood of a match with predicates describing each case. For example, the predicate value abuse is in the same verb class as attack. The service then matches all expanded predicates to a similar set of predicates that describe the problem description of each case in the repository. This is achieved using XQuery text-searching functions to discover an initial set of cases that satisfy global search constraints. Finally it applies semantic and dependency-based similarity measures to refine the candidate case set. The service returns an ordered set of analogical cases based on the match score with the query.

\subsection{Predicate matching}

Predicate expansion and matching are key to the service's effectiveness. VerbNet classes are organised to ensure syntactic and semantic coherence among members, for example the verb abuse as repeatedly treat a victim in a cruel way is one of 24 members of the judgement class. Other members include attack, assault and bully and 20 other verbs as potential expansions. The service constrains use of expansion to verb members that achieve a threshold on the degree of attributional similarity computed with WordNetbased similarity measurements (Houts et al., 1996). Given two sets of text, $T 1$ and $T 2$, the measurement determines how similar the meaning of $T 1$ and $T 2$ is scored between 0 and 1. For example, for the verb abuse, the algorithm computes the degree of attributional similarity between abuse and each co-member within the judgement class. In our example verbs such as attack, assault and bully but not honour and doubt are used to generate additional predicates in the expanded query.

\subsection{Query matching}

The expanded query is fired at situation descriptions of cases in the repository as an XQuery. The service retrieves an initial set of matched cases. It then uses the Dependency Thesaurus to refine this set using predicates relationally similar to relational predicates in the query. The service returns an ordered set of analogical cases based on the match score with the query. For example consider the Pred(j)Query = abuse (resident, resident) extracted from the sentencethe resident verbally abuses other residents at breakfast, and the Pred $(k)$ Case $=$ bully(student,child) from the sentence Ned is a sixth-grader student who constantly bullies other children. In this example all conditions for an analogical match are met: the predicate values abuse and bully are semantically equivalent whilst the object names resident and student and resident and child are not the same. According to the Dependency Thesaurus student is similar based on dependencies to resident, and children is similar based on dependencies to residents. 
Finally the attributional similarity value of resident and student is 0.25 , for resident and child 0.33 - both below the specified threshold. As a result the predicate bully (student,child) is added to the list of matched predicates for the predicate abuse (resident,resident).

Again, at the end of each invocation, the service returns an ordered set of the descriptions of the three highest-scoring cases for the app component to display to the care staff as shown in Figure 3.

\subsection{The creativity prompt generation service}

This service is used to decompose retrieved and selected case descriptions to individual creativity prompts more likely to encourage creative thinking by care staff. It decomposes the care plan enhancement part of a retrieved and selected case into constituent elements that are presented as individual creative prompts to care staff. Each prompt is of the form think about how to. The service generates the semantic content of each prompt using two different algorithms, depending on the service used to retrieve the case. Therefore, with cases in the residential care domain retrieved by the case-based reasoning discovery service, the semantic content is extracted using natural language parsing techniques to determine the first active verb of each sentence in the case. Examples of these creativity prompts include think about how to remove something from the care activities, and think about how to combine work and resources in your care activities, also shown in Figure 4.

With cases from analogical domains retrieved by the analogical matching discovery service, the service uses a different algorithm to remove terms referring to the source domain that might inhibit creative thinking by care staff. Returning to the example on the right-hand side of Figure 3, creativity prompts that make reference to the ages of drunken women and claims of innocence are likely to inhibit rather than encourage creative thinking. Instead, the service uses the attributional predicates generated by the analogical matching discovery service to generate prompts that encourage analogical transfer of knowledge using the object-pair mappings identified in each predicate. So, referring back to the analogical case retrieved from the policing domain shown in Figure 3, two creativity prompts that the service generates are think about a new idea based on the potential for violence, and think about a new idea based on the arrests.

\section{Related work}

Mobile creativity support apps are not new. Indeed, some are available online from commercial app stores. Most, however, either support non-digital creative techniques in analog form on digital devices, or more commonly provide digital support for creative methods designed originally to use non-digital artifacts. For example, the iBrainstorm app available on the Apple App Store provides users with digital post-it notes to write on and share using their mobile devices, whilst the Whack Pack app replaces the original physical pack of idea generation cards (von Oech, 1992) with digital equivalents. Most fail to provide the situation-specific creativity support provided by Carer's computational creativity services or exploit the audio channel for idea capture and reflection via play back. Moreover, most are not designed to support and change domain-specific work 
practices such as those encountered in dementia care, which in turn can limit their potential to effect changes in work practices.

Carer supports a cooperative approach to creative problem solving, similar to creativity support tools (Shneiderman, 2007). It automates information search then manipulates retrieved information to encourage cognitive creative work. The analogical matching discovery service builds on theories and models implemented in previous computational models of analogical reasoning (e.g., Greiner, 1988; Falkenhainer et al., 1989; Holyoak and Thagard, 1989), but is unusual in its integration with natural language word sense disambiguation and query expansion techniques (e.g., Stevenson and Wilks, 2001) from established information retrieval research.

Another restriction of existing analogical reasoning theories and models is the focus on single analogies that, for example, restricts Carer to exploit one case at a time. A recent resurgence of research in computational analogies is seeking to change this using an information-processing theory of creative analogies in biologically inspired design (Goel et al., 2011). The theory introduced the concept of compound analogies in which a new problem concept is generated by composing the results of multiple cross-domain analogies. Extending future versions of Carer to discover and exploit compound analogies offers an additional layer of combinational creativity not supported in the current app.

Our use of audio recording of creative ideas is inspired by NOOT, an interactive tangible system that supports (sharing of) moments of reflection during creative group meetings (van Dijk et al., 2011). When activating an interactive clips a participant in a creative process generates a time-tag in a live recording of, and during, a creative work that can be placed as relevant to provide a spatial and physical context to the audio tag, for example clipped onto a post-it note, placed on a whiteboard, or attached to a prototype. By reactivating a clip, NOOT plays back the conversation participants were having when that clip was first tagged. Carer's design was inspired by the audio capture of ideas and playback for reflective learning.

Research to introduce digital technologies into residential and dementia care is also increasing. For example, Muller et al. (2012) report that parachuting in existing technologies into residential care homes is unlikely to be effective. Rather, socio-technical themes such as sociality, trust and memory need to be considered in user-centred design processes. Our earlier use of observations, interviews and early paper and digital prototypes was consistent with this finding, and led us to apps and devices carefully adapted to fit with care working practices. Research to support people with dementia is focusing on support for relationships and maintaining a sense of self. For example, Wallace et al. (2012) report the use of digital art pieces that provide notions of home, intimacy and possessions. Our research is distinct in its use of digital technologies to support care staff rather than people with dementia. We believe that this direct support for the people who influence the quality of dementia care provided can offer greater benefits, changing the tone of the work unit to support creative problem solving as reported by Arbesman and Puccio (2001).

\section{Discussion and conclusions}

In this paper, we have reported a mobile digital app designed to support care staff to create more individualised, person-centred care for people with dementia. We believe the 
app to be novel. It is one of the first mobile apps to provide more than just resident information to care staff. It is the first that we are aware of that applies computational creativity to dementia care. In particular, we believe that Carer has the potential to offer an alternative, more cost-effective means of delivery of person-centred care. The current focus on staff training, facilitated workshops and more stringent staff selection mechanisms, although essential, are expensive in to the sector. The widespread provision of digital apps that need minimum training to use to can support the delivery of person-centred care without the costs associated with extensive human involvement.

To design the app we adopted a user-centered design process based on a detailed understanding of care work and early prototyping of creativity techniques, mobile devices and mobile apps with care staff. This led us to design an app that supports creativity techniques more likely to be effective in dementia care within the severe constraints that residential care homes impose. The app accepts assumes that all content will be expressed in unconstrained natural language accessible to care staff not trained in more restricted forms of input and output. The app, although computationally complex, runs on small devices mobile enough to carried by care staff throughout shifts. And the app is designed to encourage creative thinking in short but intensive periods between the hands-on care of residents.

We are currently trialing the version of Carer reported in this paper in residential care homes in the UK. In each home, shifts of typically 7-8 care staff have been provided with it and another app to record and communicate resident observations in digital form over periods of 4 to 7 weeks, depending on staff availability in the home. Empirical evidence from these trials indicates that, whilst the app and computational creativity services have performed effectively, and care staff found the app usable after initial periods of training, there were wider cultural and organisational barriers to longer term use of such creativity support in the homes. During a four-week trial in one residential home, seven care staff successfully used the app to generate new ideas about care and new care enhancement plans, and for at least 1 resident, applied these plans to reduce a challenging behaviour that led to increased quality of life for that resident. The staff generated the ideas primarily from cases retrieved by the case-based reasoning discovery service. However, event monitors built into the apps during the trial revealed that the care staff tended to use the app outside of their shifts, when there was time to generate and reflect on creative ideas. This has important implications for changes in working practices needed to allow creative reflective in shift-driven work such as dementia care. In contrast, during a seven-week trial in a different residential home, a similar number of care staff did not use the app for most of the trial duration. Several reasons were identified. One was a lack of management support at the shift leadership level - although the care staff used laptop computers and wall-mounted touch screen technologies to update residents' daily care records, the use of mobile devices that might intrude more into care work was resisted. Another reason was the clash that emerged between creative thinking to generate new knowledge about residents supported by the app and the prevailed care strategy in the home, which specialised in dementia care and following practices that supported and valued having full knowledge about each resident. The implicit assumption behind Carer's use - that more can be created and learned about each resident - was not consistent with the home's care management approach, and regular app use was abandoned as a result. This trial highlights the need for creativity support tools to be introduced as part of a broader, more consistent change in the home climate, work 
practices and staff training so that creative thinking will valued and rewarded. We are currently planning new trials that will roll out Carer and other mobile apps with more explicit creativity training and support, and we look forward to reporting full results from these trials in the near future.

Finally, we are able to situate our application of mobile creativity support for dementia care. We believe that our research answers the calls for creative approaches to be used in the care of people with dementia, some of which are supported with technologies. In particular, tablet technologies running apps for drawing, painting and retrieved music and film familiar to the residents are being trialed in residential homes to provide residents with dementia and their care staff with creative activities to participate together in. However, our research is the first that we are aware of that deploys computational creativity to this challenge, and if the app is successfully used in dementia care, it has the potential to counteract the negative and stressful effects that are a frequent outcome of caring for people with dementia (Help the Aged, 2007). Other reported uses of creativity in dementia care focus on more on training that everyday care - training courses in which care staff are put physically into residents' shoes (Davis, 2010), and guided exercises in which participants are encouraged to experience life mentally through the eyes of someone with dementia (Brooker, 2007]. Although Carer was not developed to deliver such training, we have made the different computational creativity services available for invocation in a virtual care home, an immersive training environment for trainee care staff to experience different forms of challenging behaviour in simulated situations. The discovery services can be invoked to retrieve new information about challenging behaviour cases that can specialise the simulated situations encountered in the virtual home, whilst creativity support available through the services can be delivered through one or more simulated characters such as senior carers. Again we look forward to reporting these new uses of computational creativity in dementia care in the near future.

\section{Acknowledgements}

The research reported in this paper is supported by the EU-funded MIRROR integrated project 257617, 2010-14.

\section{References}

Arbesman, M. and Puccio, G. (2001) 'Enhanced quality through creative problem solving', Journal of Nursing Administration, Vol. 31, No. 4, pp.176-178.

All-Party Parliamentary Group on Dementia (2009) Prepared to Care: Challenging the Dementia Skills Gap, June 2009 [online] http://alzheimers.org.uk/site/scripts/ download_info.php?fileID=735 (accessed 21 October 2010).

Alzheimers Society (2010) Statistics [online] http://www.alzheimers.org.uk/site/scripts/ documents_info.php?documentID=341 (accessed 21 October 2010).

Baldwin, C. and Capstick, A. (2007) Tom Kitwood on Dementia: A Reader and Critical Commentary, Open University Press Maidenhead, Maidenhead, Berkshire, UK.

Brill, E. (1992) 'A simple rule-based part of speech tagger', Proc., Third Conference on Applied Natural Language Processing, ACL, Trento, Italy.

Brooker, D. (2007) Person-centred Dementia Care: Making Services Better, Bradford Dementia Group Good Practice Guides, Jessica Kingsley Publishers London and Philadephia. 
Davis, R. (2010) Dementia Training Puts Care Staff in Residents' Shoe, Guardian, 2nd November 2010 [online] http://www.guardian.co.uk/society/2010/nov/02/dementia-training-care-staffantipsychotics (accessed 4 January 2011).

Falkenhainer, B., Forbus, K.D. and Gentner, D. (1989) 'The structure-mapping engine: algorithm and examples', Artificial Intelligence, Vol. 41, No. 1, pp.1-63.

Fitzpatrick, J.M. and Roberts, J.D. (2004) 'Challenges for care homes: education and training of healthcare assistants', British Journal of Nursing, Vol. 13, No. 21, pp.1258-1261.

Gentner, D. (1983) 'Structure-mapping: a theoretical framework for analogy', Cognitive Science, Vol. 7, No. 2, pp.155-170.

Goel, A., Vattam, S., Helms, M. and Wiltgen, B. (2011) 'An information-processing account of creative analogies in biologically inspired design', Proceedings 8th ACM Conference on Creativity and Cognition, ACM Press, pp.71-80.

Greiner, R. (1988) 'Learning by understanding analogies', Artificial Intelligence, Vol. 35, No. 1, pp.81-125.

Help The Aged (2007) My Home Life: Quality of Life In Care Homes; A Review of the Literature London [online] http://myhomelifemovement.org/downloads/mhl_review.pdf (accessed 5 January 2011).

Higgins, J.M. (2006) 101 Creative Problem Solving Techniques: The Handbook of New Ideas for Business, New Management Publishing Company, Inc., Florida, p.142.

Holyoak, K.J. and Thagard, P. (1989) 'Analogical mapping by constraint satisfaction', Cognitive Science, Vol. 13, No. 3, pp.295-355.

Houts, P.S., Nezubd, A.M., Magut Nezubd, C. and Bucherc, J.A. (1996) 'The prepared family caregiver: a problem-solving approach to family caregiver education', Patient Education and Counseling, Vol. 27, No. 1, pp.63-73, retrieved from Elsevier.

Innovation Company (2002) 'Sticky Wisdom (?WhatIf!)', Capstone Publishing Company Limited, Chichester.

Kipper, K., Dang, H.T. and Palmer, M. (2000) 'Class-based construction of a verb lexicon', AAAI-2000 Seventeenth National Conference on Artificial Intelligence, Austin, TX, USA.

Karlsen, K., Zachos, Z., Maiden, N.A.M., Jones, S., Turner, I., Rose, M. and Pudney K. (2011) 'Supporting reflection and creative thinking by carers of older people with dementia', to appear in proceedings 1st International Workshop on Pervasive Care for People with Dementia and their Carers, Dublin, Ireland, May 2011.

Knapp, M. and Prince, M. (2007) Dementia UK: A Report Into the Prevalence and Cost of Dementia', prepared by the Personal Social Services Research Unit (PSSRU) at the London School of Economics and the Institute of Psychiatry at King's College London, for the Alzheimer's Society.

Le Storti, A.J., Cullen, P.A., Hanzlik, E.M., Michiels, J.M., Piano, L.A., Lawless, R.P. and Johnson, W. (1999) 'Creative thinking in nursing education: preparing for tomorrow's challenges', Nursing Outlook, Vol. 47, No. 2, pp.62-66.

Levin, B. (1993) English Verb Classes and Alternations: A Preliminary Investigation, University Chicago Press, Chicago.

Lin, D. (1998) 'Automatic retrieval and clustering of similar words', in COLINGACL, pp.768-774.

MacManus, J. (2011) Creative Problem Solving for Dementia Care Staff, MSc Dissertation, School of Informatics, City University London, January 2011.

Machin, S. and Wilson, J. (2004) 'Minimum wages in a low-wage labour market: care homes in the UK', The Economic Journal, 114, March, C102-C109, Royal Economic Society 2004, Blackwell Publishing Oxford.

McCarthy, D., Koeling, R., Weeds, J. and Carroll, J. (2004) 'Using automatically acquired predominant senses for word sense disambiguation', Proceedings of the ACL 2004 Senseval-3 Workshop Barcelona, Spain. 
Michalko, M. (2006) ThinkerToys: A Handbook of Creative-Thinking Techniques, 2nd ed., Ten Speed Press, New York, pp.132-136.

Miller, G.A., Beckwith, R., Fellbaum, C., Gross, D. and Miller, K.J. (1990) 'Introduction to WordNet: an on-line lexical database', International Journal of Lexicography, Vol. 3, No. 4, pp.235-244.

Muller, C., Neufeldt, C., Randall, D. and Wulf, V. (2012) 'ICT-development in residential care settings: sensitizing design to the life circumstances of the residents in a care home', Proceedings ACM CHI'2012 Conference, ACM Press, pp.2639-2648.

Osborn, A. (1965) 'The creative trend in education', Source Book For Creative Problem Solving: A Fifty Year Digest of Proven Innovation Processes, Creative Education Foundation Press, New York, Selection 4.

Owen, T. and Meyer, J. (2009) 'Minimizing the use of 'restraint' in care homes: challenges, dilemmas and positive approaches', Adult Services Report 25, Social Care Institute of Excellence [online] http://www.scie.org.uk/publications/reports/report25.pdf (accessed 1 December 2012).

Salton, G., Wong, A. and Yang, C.S. (1975) 'A vector space model for automatic indexing', Communications of the ACM, November, Vol. 18, No. 11, pp.613-620.

Shneiderman, B. (2007) 'Creativity support tools: accelerating discovery and innovation', Communications of the ACM, Vol. 50, No. 12, pp.20-32.

Sternberg, R.J. (Ed.) (1999) Handbook of Creativity', Cambridge University Press, New York.

Stevenson, M. and Wilks, Y. (2001) 'The interaction of knowledge sources in word sense disambiguation', Computational Linguistics, Vol. 27, No. 3, pp.321-349.

Stickdorn, M. and Stevenson, M. and Wilks, Y. (2001) 'The interaction of knowledge sources in word sense disambiguation', Computational Linguistics, Vol. 27, No. 3, pp.321-349.

van Dijk, J., van der Roest, J., van der Lugt, R. and Overbeeke, K. (2011) 'NOOT: a tool for sharing moments of reflection during creative meetings', Proceedings 10th ACM Creativity and Cognition Conference, Atlanta Georgia, Nov 2011, ACM Press.

von Oech, R. (1992) Creative Whack Pack [Card Deck] Stamford, US Games Systems.

Wallace, J., Thieme, A., Wood, G., Schofield, G. and Olivier, P. (2012) 'Enabling self, intimacy and a sense of home in dementia: an enquiry into design in a hospital setting', Proceedings ACM CHI'2012 Conference, pp.2629-2638, ACM Press.

Wilks, Y. and Stevenson, M. (1996) 'The grammar of sense: is word-sense tagging much more than part-of-speech tagging?', Sheffield Department of Computer Science, Research Memoranda.

Wimo and Prince (2010) [online] http://www.alz.co.uk/research/worldreport/ (accessed 10 July 2012).

Zachos, K. and Maiden, N.A.M. (2008) 'Inventing requirements from software: an empirical investigation with web services', Proceedings 16th IEEE International Conference on Requirements Engineering, IEEE Computer Society Press, pp.145-154.

Zachos, K., Maiden, N.A.M., Zhu, X. and Jones, S. (2007) 'Discovering web services to specify more complete system requirements', Proceedings CaiSE'2007, Springer-Verlag Lecture Notes on Computer Science LNCS 4495, pp.142-157. 